\title{
The Earth in space: An essay on the origin of the Solar system
}

\author{
SiHBDis BuRMin
}

Ricevuto il 20 Giugno 1963

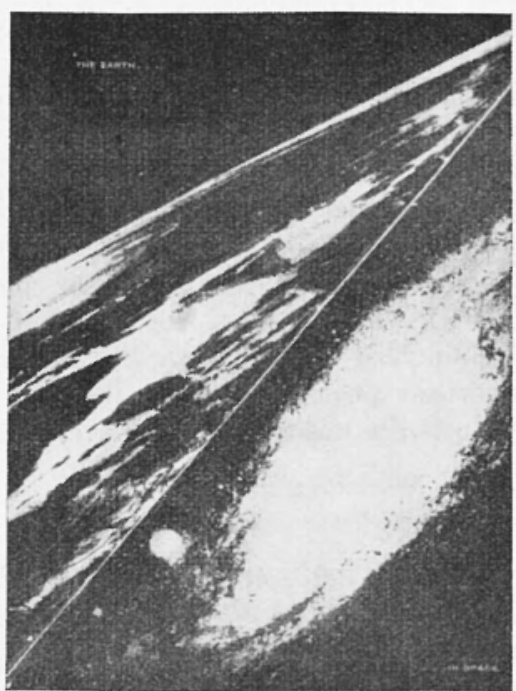

"Reproduced by kind permission of Prof. R. H. Diclie, Princeton University, d: Editor, Science; Science, 138, n. 3541.9. pages 654-664 (1962)".

"... Science is always most completely assimilated when it is in the nascent state".

J. C. Maxweli.

'You couldn't deny that, even if you tried with both hands .

'I don't deny things with my hands,' Alice objected.

'No body said you did,' said the Red Queen. 'I said you couldn't if you tried'.

"She's in that state of mind,' said the White Queen, that she wants to deny something-only she doesn't know what to deny'.

'A nasty, vicious temper,' the Red Queen remarked.

Lewis CarroLi, Through the Looking-Glass. 
SUMmary. - The origin of the sum and planets has been reviewed from manifold considerations - nuclear, astrophysical, chemical and geophysical. Basically, there are two schools of thought: monistic, which postulates that the sun and the planets formed from some primordial system of gases; and dualistic, which holds that the planets and meteorites had genesis in the sun's collision wtilh another star. 'The extreme improbability of collision almost discards this hypothesis.

The present day accepted theories are, hence, the monistic ones, and the one particularly favored is the Dust - cloud hypothesis - that the sun condensed into a star due to the gravitational collapse of a massive interstellar gas-cloud, and subsequently gave birth to planets as further evolution of the clond progressed. Studies of extinct radioactivities, within the framework of the above hypothesis, give clue to the early history of the solar system and in particular indicate that the time interval between the start of condensation and the formation of the meteorite parent-bodies is less six million years (Cameron). In this context the origin of stars from "globules" or proto-stars has been briefly diseussed.

A somewhat "exotic" theory of the formation of planets from the sun which hinged on the concept of secular decrease of the 'constant' of gravitation with the age of the universe (Dirac's hypothesis) has been discussed. The earth (with expansion of its volume) and other celestial bodies might provide empirical confirmation of the concept of diminishing gravitation - an important problem of general relativity. This new idea of physies might revolutionise fundamental concepts in geology and geophysies.

Riassunto. - L'origine del sole e dei pianeti e stata riesaminata sulla base di molteplici considerazioni - nucleari - astrofisiche, - chimiche e geofisiclie. Fondamentalmente ci sono due scuole di pensiero: monistica, che postula che il sole ed i pianeti si formano da alcuni primordiali sistemi di gas; e dualistica, che ritiene che i pianeti e le meteoriti hanno avuto origine dalla collisione del sole con altre stelle. L'estrema improbabilita di collisione rende questa ipotesi quasi nulla.

Le teorie accettate oggi sono, percio quelle monistiche e particolarmente favorita e la ipotesi della nuvola di polvere - che il sole, cioe, si sia condensato attraverso processi gravitativi a cui la nebula primigenia fu soggetta e di conseguenza abbia dato vita ai pianeti con processi evolutivi variamente interpretati. Nel qualro delle ipotesi di cui sopra, studi su radioattivita spente danno indicazioni sulla storia recente del sistema solare ed, in particolare, indicano che l'intervallo di tempo tra il principio del condensamento e la formazione dei corpi generanti le meteoriti, e inferiore a sei milioni di anni (Cameron). In questo lavoro viene brevemente discussa l'origine delle stelle dai "globules " o proto-stelle.

E stata esaminata una teoria alquanto "ecentrica" sulla formazione dei pianeti dal sole, che si imperniava sul concetto della diminuzione della costante di gravitazione con l'eta dell'universo (ipotesi di Dirac). I a terra con espansione (lel suo volume) ed altri corpi celesti possono dare una con- 
ferma empirica del concetto di diminuzione di gravitazione - un problema importante di relativita generale. Questa nuova idea della fisica, potrebbe rivoluzionare concetti fondamentali di geologia e geofisica.

1. The problem of the origin of the Solar System is one of the three most important ones in natural history - the other two being the origin of life on earth and the descent of man. It is also one of the oldest unsolved enigmas of scientific philosophy and has attracted masterminds of both science and philosophy in each century since the time of Scientific Revolution. Scientific cosmogony begins with the vortex theory of Descartes even before Newton discovered the law of gravitation. In the past two and a half century a galaxy of inquisitive spirits were fascinated to this problem: Kant, Laplace, Milne, Jeans, Jeffreys, Kuiper, Hoyle, Urey, Weizsacker, Schmidt and others. In 1950 Ter Harr has reviewed and variously criticised all of the then existing theories. And now within the last decade many more speculations have appeared; their numbers seem to be increasing with the rising interest in the space researches and with ever brightening hopes of man's conquest of outer space.

Our knowledge of the physies of interstellar space has now at last come to a stage that we may formulate realistic theories of star formation. These theories have a bearing on the problem of origin of Solar system in that most of them postulate the formation of the sun and the planets together. The present day accepted theories have a common theme in that all postulate an interstellar gas cloud that is massive enough for a gravitational collapse to take place leading to star formation. However, before going into their discussions, some of the conspicuous regularities of the Solar system will first be presented. These characteristics will show that the Solar system possibly could not have been formed by chance.

Ter Haar (1948) arranged them in the following orders:

Group A. Regularity of the orbits. The planetary orbits are almost circular, lie in one plane and revolution is in one direction, with the sun rotating in the same direction - there is a "one-way traffic" in the Solar system. The equatorial plane of the sun is near to the plane of the orbits.

Group B. Regularity in the planetary distances. The distance of the planets follow some general pattern of which Titus-Bode's rule is 
one. The radius of each planetary orbit is roughly twice as large as that of the orbit nearest to it in the direction of the Sun, or mathematically the distance from the Sun to the other planets is expressed by the formula

$$
x=0.4+0.3 \times 2^{n} \text { A.U. (Astronomical Units) }
$$

where $n$ is the number of the planets (for Venus, $n=0$, for the Earth $n-1$, etc.), and 0.4 (in A.U.) is the distance of Nercury from the Sun (Fig. 1).

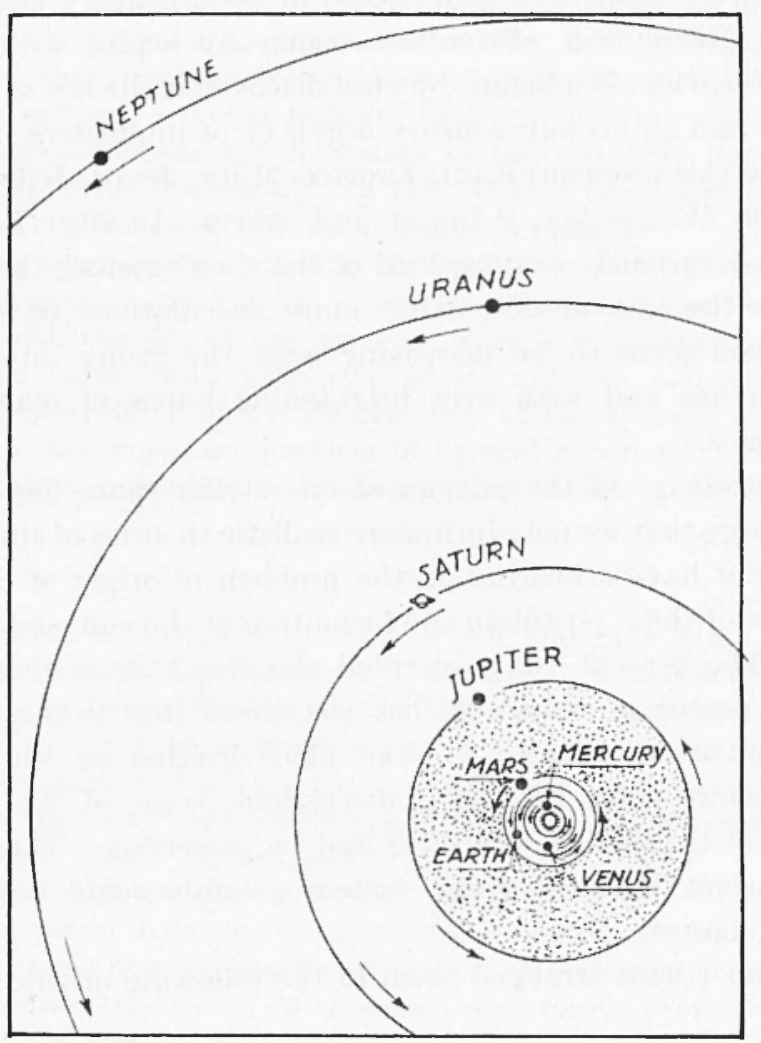

Fig. 1

(after A. Oparin \& V. Fesenkov, 1960).

Group $C$. The division of the planets into two sharply distinet groups: the inner planets, Mercury, Venus, the Earth and Mars, are comparatively small but possess great density, rotate quite slowly around their axes and have a small number of satellites, and the outer planets - Jupiter, Saturn, Uranus and Neptune, are big, have lower 
density, great speed of rotation and numerous satellites. (The planet Pluto is on the fringe of the system and may not conform to the same regularities).

Group D. The distribution of the angular momentum: although the Sun possesses more than $99 \%$ of the total mass of the Solar system it accounts for less than $2 \%$ of the momentum, the remainder being accounted for by the planets.

The above groups of features are basic and any theory of planet origin must explain them. These regularities strongly suggest that the system as a whole was formed by a single process involving the Sun. Some support for this view comes from the measurement of the ages of the Earth and the Sun. From Uranium-Lead radionctive age determination method the Earth's age is 4.5 or 4.7 billion years. From considerations of stellar structure and stellar evolution, the Sun's age is 5 to 8 billion years. This is also the age of many stars and star clusters of our Galaxy. Again Russel, from spectro-chemical analysis of the solar atmosphere concluded that the distribution of the widespread elements is almost exactly the same on the Sun as it is on the Earth. This may be regarded as proof of the common origin of the Sun and the planets by which it is surrounded.

2. All theories of planetary origin can be divided into two groups. Those in one group are based on the assumption that the system has been formed as a result of an interaction between the Sun and another celestial body, causing a catastrophit change. These theories may be ealled cataclysmic or dualistic. The theories of the second group suppose that the system has been formed by the slow evolution of a primordial system. These theories may be called monistic. (H. S. Jones, 1956).

Planetary systems must be much more abundant on the basis of slow evolution of primordial system than if some catastrophic change is necessary to produce them. If we could detect planetary systems around stars and estimate their relative frequencies, we could then form a verdict in favour of the one or the other.

Among the stars about $80 \%$ (according to Gamow) are known to be multiple stars - chiefly binaries. It has been argued that the formation of a planetary system may be a special case of the process of the derelopment of multiple star systems. From consideratious of mass - ratios in binary systems, Kuiper (19501) has estimated that the 
probability of the mainsequence star like the Sun in having a planetary system is about $10^{-2}$ or $10^{-3}$. If this is true, then all dualistic theories of the formation of the Solar system must be discarded, as the extremely rare chance encounter's of two stars on which they all rest cannot explain so many planetary systems.

The dualistic theorr is historically probably the first one proposed by Buffon (1719). He saw the origin of the planetary system as a result of a collision between the Sun and a comet that ame from the depth of interstellar space. Following the poetic word - picture of Gamow, it was a "romete fatale" with a long brilliant tail brushing the surface of our, at that time lonely, Sun, and tearing from its giant body a number of small "drops", which were sent spinning into space by the force of the impact.

Then came the monistic theory of Kant-Laplace. They conceived of the whole Solar spstem as originating in a condensation of a large mass of diffuse gas. (Kant's hypothesis was one of the first to consider the process of development of the cosmic bodies, which were until then believed to have been immutable from the very moment of their supposed "creation". Judging now in retrospect it was a fore-rumer of the theory of evolution in biology as conceived by Darwin, Wallace and others, which has now become a living doctrine in multiple disciplines of science). If this mass had a very slight rotation, however small, the process of contraction would cause the angular velocity to increase. There would come a time, however, when the gravitational attraction of the central parts of the proto-Solar system would be unable to retain the rapidly moving outer parts in circular orbits - the centrifugal forces of rotation would become important compared to the gravitational and would result in the ejection of a series of gaseous rings along its extended equator. The rings formed in this way were supposed to have broken up later and to have condensed into various planets circling at different distances around the Sun. This hypothesis accounted qualitatively for the main regularities in the Solar system.

But there is a fatal objection to this hypothesis. It is unable to account for the distribution of the angular momentum in the Solar system. It can be shown that in any system contracting under its self-gravitation, each unit mass of material would carry exactly the same amount of angular momentum as any other unit mass, thus it is a failure to explain the angular momentum distribution.

To make escape from the fundamental difficulties of Kant-Laplace hypothesis, the dualistic theory was revived by Jeffrey and Jeans. This ti- 
me the Sun and a passing star were supposed to collide. In order to account for almost circular orbits of the planets, it was postulated that at that time the Sun was surrounded by a uniformly rotating gaseous envelope, which helped to turn the originally elongated planetary orbits into regular circles by viscous drag. The gaseous envelope gradually dissipated into interstellar space, and the faint luminosity known as Zodiacal Light is all that is left of the "past glory". However, Tolke has calculated that for the planet to reduce the eccentricity of its orbit from, say, 0.5 to 0.1 , it had to absorb five times its own mass from the medium. In other words, the resisting medium becomes a feeding one, and the hypothesis is untenable.

Jeans' theory also does not solve the angular momentum difficulties. The consideration of the physical condition of the ejected material leads to a further difficulty, pointed out by Russel (1935), which is very serious. When the Sun met with a near encounter with another star, its temperature must have been more than a million degrees and its pressure more than a million atmospheres. The mean velocity of hydrogen will be $100 \mathrm{mps}$. and would rapidly dissipate into space inspite of gravitational attraction of ejected mass; the remainder of the mass will, however, form an extended gaseous nebula around one or of the stars involved.

As the catastrophic-type hypothesis could not give a satisfactory solution to the problem of the origin of the Solar system, the pendulum was again swung to the monistic type i.e. origin by gradual evolution of a primordial system. Such theories all start from the assumption that the Sun was at the centre of a diffuse gaseous cloud, which may have been an interstellar cloud into which the sun has entered, or a gaseous cloud from which the sun has condensed. The dark globules which are abundant in the Milky-Way regions are believed to be "proto-stars", where stars are in the process of condensing out of a gaseous system. Alfven divides theories of this type into two classes, according to whether the Solar system has originated from a cold gas or hot ionised ones.

The Russo-German astronomer Academician Otto Schmidt has developed a theory of the origin of Solar system of cold type, br the Sun's capture of an interstellar gas-dust cloud aimlessly wandering in the vast expanse of the Milk-Way. The planets were formed by the agglomeration of a large number of solid matter each moving around the Sun in its own independent elliptical orbit. When large numbers of bodies were joined into a single planet their orbits were, naturally, averaged, and as a result, they could only be fully symmetrical, i.e. circular, and close to a plane perpendicular to the vector of the 
principal angular momentum of the whole system. The co-planar and co-directional motion of the planets result from the averaging of the angular momenta of many bodies. The problem of the distribution of angular momentum does not arise here, for the Sun could acquire from the Galaxy material possessing sufficient momentum. Schmidt also derived a law of planctary distance that bear close resemblance to Bode's law. In many ways his theory is the most highly developed of those in the field. The main requirement in Schmidt's theory would seem to be that the relative velocity of the Sun and the cloud must be rather small, not exceeding one $\mathrm{km} / \mathrm{sec}$. On this theory planet formation is not uncommon at the late stage of Galactic evolution.

In accord with Schmidt's idea of planetary formation from cold circum-solar gas-dust cloud, is the German cosmologist C. ron Weizsacker's (1943) theory, which is in essence an improvement of the original Kant-Laplace hypothesis. The starting assumption is that the Sun was at the center of a revolving gas-dust cloud having its mass $\approx 0.1 \mathrm{冫}$. The resultant of different particles moving round the Sun in independent orbits was a circular equatorial envelope due to frictions and visenous drags. Viscuous forces tending to equalise the angular velocities within the disk slowed down the faster moving inner parts and speeded up the pace of the slower moving outer parts. It is easy to see that this process makes the inner parts gradually transfer angular momentum to the outer rims. Escape of the gases from the outer parts to the intersteller space carries away some of the angular momentum.

Making a greater use of thermodynamics and statistical physics than before, Weizsacker studied how semi-stationary states could be formed round the Sun. It will be presently shown with numerical details that such a revolving disk will form violent turbulance, the Reynold's number $R$ being $\approx 10^{11}$, much bigger than $R \simeq 10^{3}$ for lamilar flow. This can be verified by substituting the numerical values in $R=\varrho v d / \eta$ where $\varrho$, the mean density $\approx 10^{-10} \mathrm{gm} / \mathrm{cm}, v$, the average velocity $\approx 10^{5} \mathrm{~cm} / \mathrm{sec}, d$, a length taken upto the distance of Saturn and of an approximate order of magnitude of the extent of planetary system $\approx 10 \mathrm{~A} . \mathrm{U} .=1.5 \times 10^{14} \mathrm{~cm}$, and $\eta$, the coefficient of viscosity $=10^{-2}$ poise. Such huge turbulance would cause the material form vortices. Weizsacker next showed that a series of annuli rotating round the sun and each containing five vortices would form a stable arrangement. The radii of the annuli approximately follows Bode's empirical law. 
Due to large viscuous stresses which set up between adjacent vortices farour formation of small eddies between the rings. These secondary vortices acted as more suitable platforms for the condensation of matter, forming proto-planets. Separate condensations in each rortices within an annular ring gradually came near each other and formed a single planet.

The condensation process gives a distribution of mass found actnally. The only serious disagreements are that the mass of Mars is much smaller than which it should have been. Even if we assume that the Moon was formed alongwith Mars in the same ammular ring, but before joining Hars to form a single planet it was captured by the Earth, it does not greatly solve the problem. Also, the theory requires a planet between the Mars and Jupiter where at present asteroid belt is found, but the absence of any such planet was due to non-operation of solid accretions (according to the latest soviet researches on meteorites from this zone) being disturbed by the gravitational pull of the giant planet Jupiter.

Weizsacher's above theory got recognition from western scientists including ter Harr, and the Indian scientist Chandrasekhar and others tried to further develope it. It was critised by Soriet scientists, e.g. by Levin, possibly because they are influenced by the work of Academician Schmidt, who also as we have seen formulated the evolution of a cold gas cloud. A subtle dilemma in Weizsacker's theory, moreover, arises because it fails to account for the narrow range of specific binding energies $\left(10^{12}-10^{16} \mathrm{erg} / \mathrm{gm}\right.$.) in astronomical systems; from galaxy (lusters, galaxies, multiple stars, stars, solar system to planets and satellites. (Star clusters, however, have gravitational binding energy per unit of mass in the range $10^{10}-10^{12} \mathrm{erg} / \mathrm{gm}$.). However, for the importance of the turbulence in the above theory its extensive mathematical theory is carried out by Taylor, von Karman, Kolmogoroft and W. Heisenberg.

A modified form of Weizsacker's theory is due to Kuiper. He applied Kolmogoroff's turbulence spectrum derived for non-rotating media, and comes to the conclusion that the repeated formation and dissolution of these eddies would give rise to small condensations, and not a few of large ones as Weizsacker supposed. The only way to escape is if gravitation helps some of the large vortices for massive condensations to being. Further if, $A$ denotes the distance between the centre ot two planetary masses whose mean distance from the Sun is $a$, then according to Kuiper, the planetary masses will be proportional to $(\Delta / a)^{3}$. 
He finds this result nearly satisfied by the actual planetary masses and distances. This empirical relation also holds good for the satellite systems.

A lot of systematic works have been done on the orbital and other characteristies of close binary system in the galaxy. One unexpected and exciting result that has emerged is Kuiper's deduction that the Solar system is a degenerate double star in which the second star did not condense into a single star but was spread out, and formed planets and comets (Kopal, 1959). WVe shall deal with this question further towards the end.

So far we were absorbed with celestial dynamics to trace the mechanism of planet formation. The role of electromagnetic forces in this phenomenon was first underlined by Alfven (1954), and it has since then assumed paramount importance as may be judged from the remark of a scientist that features which were formerly attributed to deities are now assigned to electromagnetism! Alfven postulated a Sun condensed from a magnetic cloud of gas which possessed a strong magnetic field. The residual gaseous cloud which contained mannetic field formed "plasma" due to radiation from the Sun. The ionised gas was prevented from falling into the sun by its manetic field as well as that of the Sun, whereas the un-ionised gases fell into the Sun. With increasing distance from the Sun, the amount of ionisation progressively decreased and the chemical composition of the gas falling in towards the Sun varied as the distance from the Sum, depending on their ionisation potentials. As the clonds fell, their speed increased and on collision with the permanent ionised layer around the Sun, further ionisation of cloud material could oceur. The magnetic field began to act as a brake as ionisation commenced, and eventually stopped any further fall at distances according to their ionisation potential, and the ionised gases went into orbit around the sun. The transfer of angular momentum from the more rapidly spinning Sun to the continuously receeding and less rapidly revolving gas condensations was magneto-hydrodynamical in nature.

An interesting outcome of Alfven's theory is concerning the origin of the Moon. Following his suggestion, the "A" cloud (having highest ionisation potential) was stopped $2.25 \times 10^{13} \mathrm{~cm}$ from the Sun, i.e., about the present distance to Mars and from it condensed Mars and the Moon by accretion of dust condensed from the cloud. The "B " rloud having different composition and velocity (and lesser ionisation potential than $A$ ), ionised and began condensation at $1.09 \times 10^{13} \mathrm{~cm}$ from which 
were formed Earth, Venus and Mercury. Due to overlap between the regions newly formed, Moon was captured by the Earth. This is in tune with the fact that the Moon is not in the main gravitational field of the Earth. Alfven's original theory or its variation based essentially on the behaviour of plasmas shall receive benefits from both the theoretical and experimental researches undertaken in plasma physics for its importance both in astrophysics and in human welfare ( $\mathrm{f}$. researches in controlled thermo-nuclear fusion reactions in some big laboratories of the world).

The basic assumption of Alfven is that the early Sun was highly magnetic (thousands of time more intense than it is now). The soriet astronomer Leving for this reason calls the theory arbitrary, but the author feels to reserve his comment till a unique rerdict is arailable from studies of structure and evolution of stars. It may, howerer, be pointed out that some stars like $T$ Tauri which are believed to be undergoing at present early stellar erolution are highly magnetic. It is not therefore impossible that the primitive Sun was highly magnetic as Alfren believes.

Urey (1957) has examined the boundary conditions for the origin of Solar system from chemical considerations. He concludes that there is at least a possibility that solid particles rather than gases dominated the processes. On the oceasion of the Silliman lecture in 1951, Urey made an attempt to inchde chemical evidences within the framework of ron Weizascker-Kuiper theory.

3. However, nuclear and astrophysical evidences discussed below, brings forward the indequecy of Kuiper"s model of large proto-planets; and we shall analyse the problem of origin of the Solar system viewing from the broader perspective of cosmology. Studies ean be made of the contraction of a cosmic gas cloud due to its gruritational instability by means of Jean's instability criteria or with the aid of the ririal theorem. Following the later approach, cameron (1962) deduced the minimum mass necessary for a cloud to contract is

$$
M=\frac{61, \mathrm{C} 00}{\left(n_{\mathrm{H}} 1 / 2\right)} M \supset ;\left(M \odot=\text { mass of the Sun }=1 \cdot 985 \times 10^{33} \mathrm{gm}\right)[1]
$$

where $n_{\mathrm{H}}$ is the no. of hydrogen atoms per c.c., e.g. clouds of 3000 Solar masses can contract if $n_{\mathrm{F}}=400$, which is not too uncommon in the 
interstellar medium (Davies, 1958). It is interesting to speculate about the conditions that may be necessary to cause an interstellar cloud to collapse. It can be induced if a large pressure say, stellar radiation

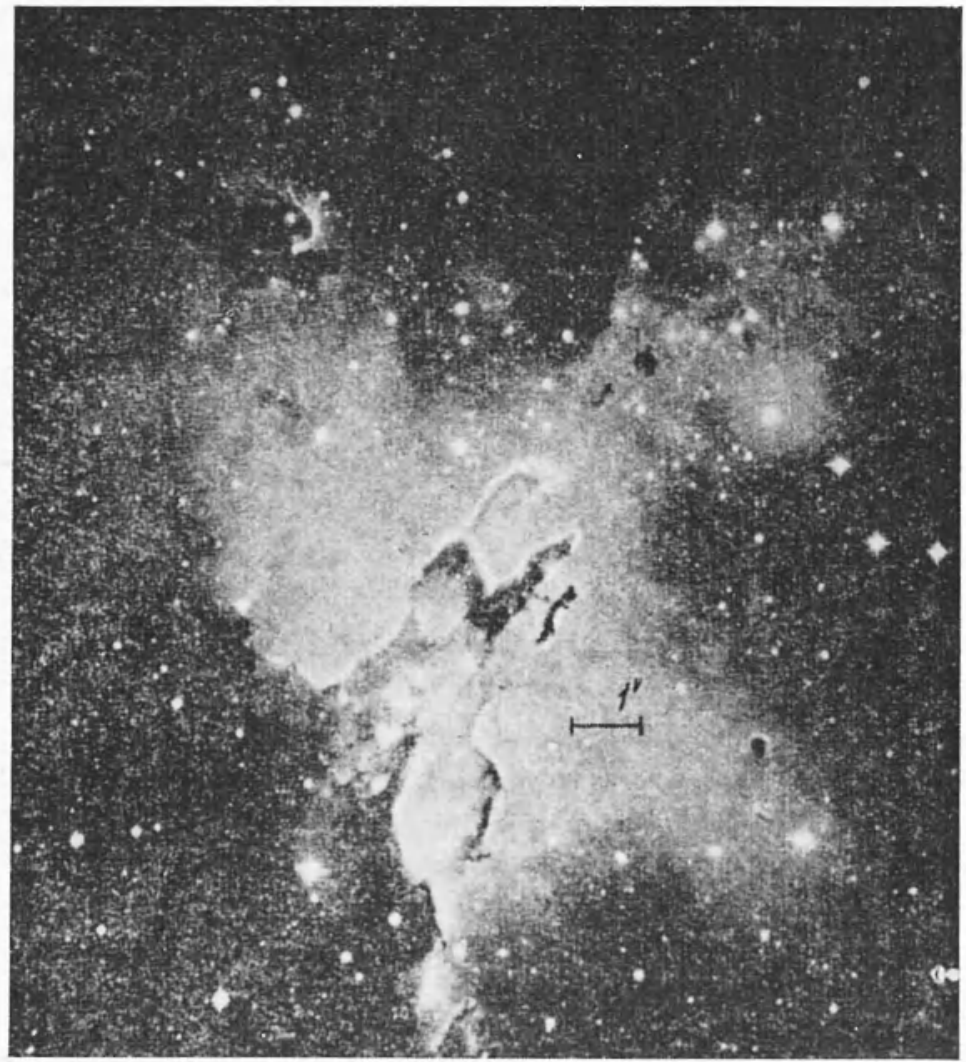

Fig. 2 - A detail of nebula M 16, photographed with the Momnt Palomar 200 in. reflector. Dark gloubules, bright rims and an elephant 'trunk" are visible. The matter is made luminous by the hot young stars embedded in it. At the present time stars are probably being formed in such regions. The grobules - the small dark patches of dense matter - may represent an early stage in the formation of stars.

or cosmic ray pressure is exerted on the boundary of the cloud. Since dense clouds occur in a region of the interstellar medium where much star formation is going on, the formation of new $O$ and $B$ Stars (*) may

$\left.{ }^{*}\right)$ The spectral classification of main-sequence stars are in order: $O, B, A, F, G, K, d I$ and $R, N . S$. (so easy to remember: "Oh, Be $A$ Fine 


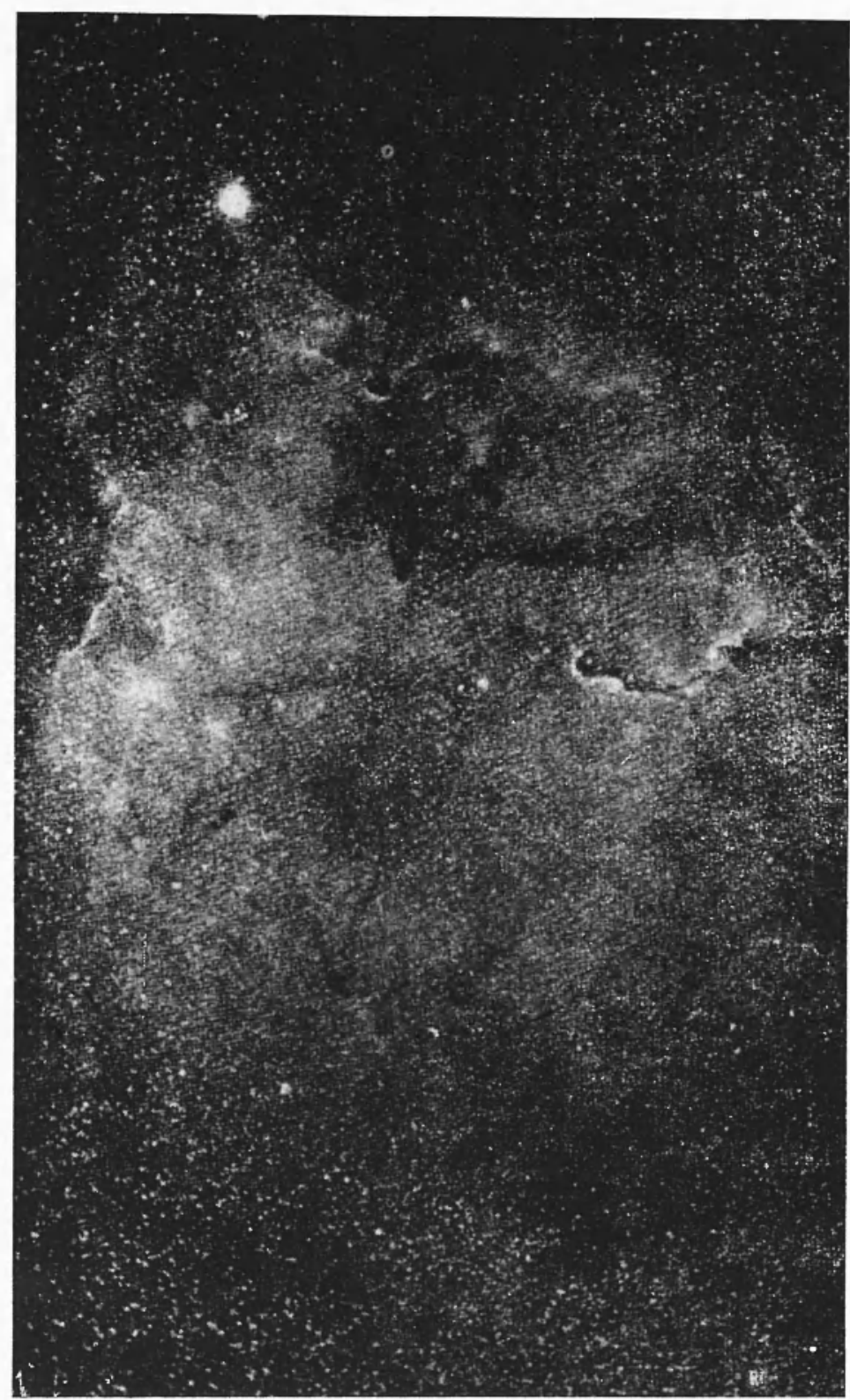

Fig. 3 - Nebula IC 396 in the constellation Cepheus, showing dark globules. 
cause the oceasional ionization of the hydrogen survounding the boundary of dense clouds, thus producing FI II $\left({ }^{*}\right)$ regions which may exert sufficient pressure depending upon temperature on the boundary of a cloud to cause its collapse. Lebedinsky has also suggested similar mechanism to compress globules to high density. Globules are regarded as possible embryonice stars: they are small, round, very dense clots which are ofien visible in the background of nebulate (Figg. 2. 3). Another mechanism is supernova explesion which mar precipitate general collapse by affecting an orerall surface pressure. The massive stars form Type II Supernova $\left({ }^{* *}\right)$ are also those that begin life as 0 and $B$ star's. Thus the two processes may operate together.

Side by side with light pressure another factor may operate to initially induce small condensations to start with before gravitation assumes the learlership. This is turbulance in interstellar space. These can easily produce rudimentary aceretion in the primordial gas (Gamow 1952). Harlow Shapley contoured the distribution of galaxies in celestial hemisphere; it is divided into large whirls and suggest eddies. Recent; observational evidences, including that of radio astronomy, also point to turbulance in interstellar space (Camcron, 196\%).

As the cloud contracts, according to equation [1] the minimum mass required to have a gravitationally stable configuration rapidly diminishes. Thus it becomes possible for the cloud to fragment into subcondensation. The process of mass ejection during condensation is very common in some stars, for example Fig. 4 shows the instance of $B$ Lyrae star emitting a powerful stream of gas which partly envelops the satellite and partly enters the common gas ring surrounding the whole system. The frugmentation process has been discussed by Foyle and by Mestel and Spitzer. They conclude that fragmentation will continue until the fragments become too opaque to their own radiation, and this will occur for bodies of roughly Solar mass.

Girl, Kiss Me Right Now, Sweet!"). The hottest stars (surface temperatures of $25.000^{\circ} \mathrm{K}$ up) are of Spectral type $O$, followed by $B$ stars $\left(25.000^{\circ} \mathrm{K}\right.$ $\left.-11.000^{\circ} \mathrm{K}\right)$ and so on.

(*) H II is the name given to hot, ionised hydrogen; the cool, unionised hydrogen atom is called $\mathrm{H}$ - I.

(**) Supernovae are gigantic nuclear stellar explosions in which the whole star is blown up. It is believed to herald the death of the evolutionary life of a star. Type II is the more intense of the two types the supernovae explosions may be classified into (after Cameron, 1962). 
Recent studies in cosmology have proved both theoretically and experimentally the presence of interstellar magnetic field $\left(H \approx 5 \times 10^{-8}\right.$ gauss) (Davies 1962). The presence of this brings the question how effective it may be in transferring angular momentum from the contracting cloud to the surrounding disk or interstellar medium. This problem has been considered by Ebert (1960) who considered a magnetic field parallel to the axis of rotation of the cloud which transmitted a

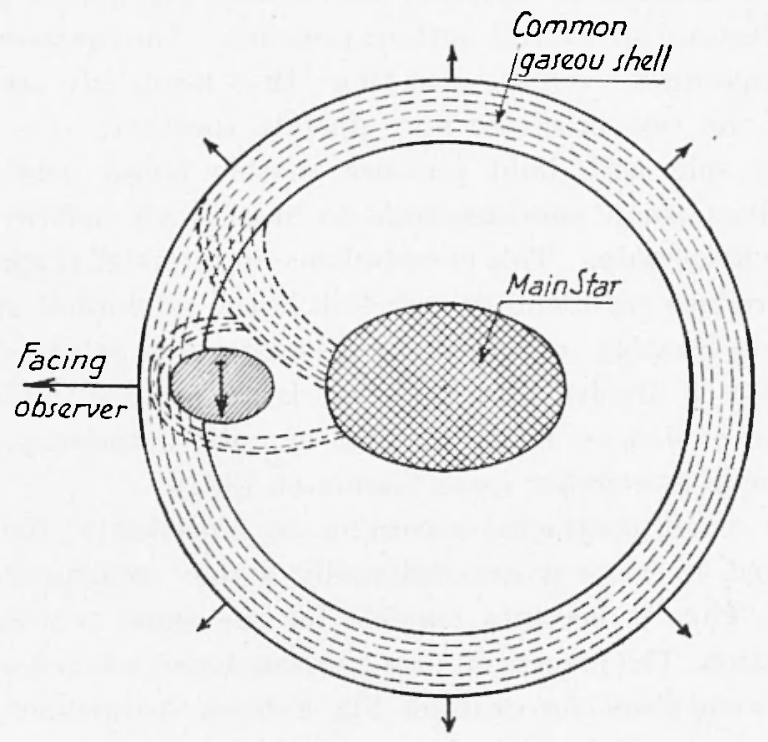

Fig. 4

(after A. Oparin \&V. Fesenkov, 1960)

torque to the surrounding interstellar medium as it was twisted due to rotation. A plausible magnetic configuration is shown in Figures 5 and 6. Hoyle (1960) finds for a convective Sun (as all dwarf stars are) the angular momentum transfer is very efficient, while Cameron (1962) derives the twist of the cloud and the magnetic lines of force to be very small, and suggests that not much angular momentum can be transmitted from the contracting cloud to the surroundings during the period of contraction. Evidently this problem deserves very careful investigation.

When material leaves a rotationally unstable star it is very likely that a disk is formed in the equatorial plane. There is observational estimate by Underhill of a star Pleione that the rotation velocity of the star is larger than that of the disk. Hence magnetic lines of force which originally connected the star with the disk naturally become twisted into a spiral 
structure. Two cases next arise depending on if the turns of the magnetic lines of force can mainly be stored inside the star, or they have to be mainly stored outside the star. In the former case, the disk can move

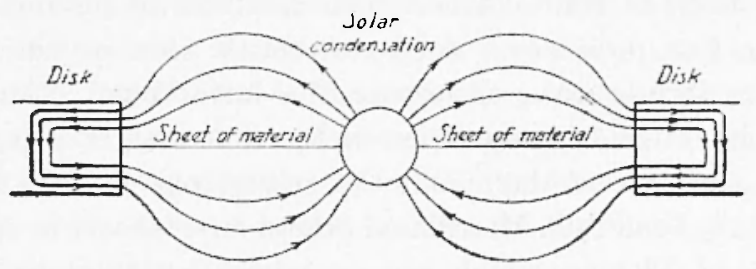

Fig. 5

(after Hoyle, 1960).

about the star for a longer time, and acquires angular momentum at the expense of the star, and thus receeds from the centre. A gradual evolution of one such primordial disk may have led to the origin of the Solar system.

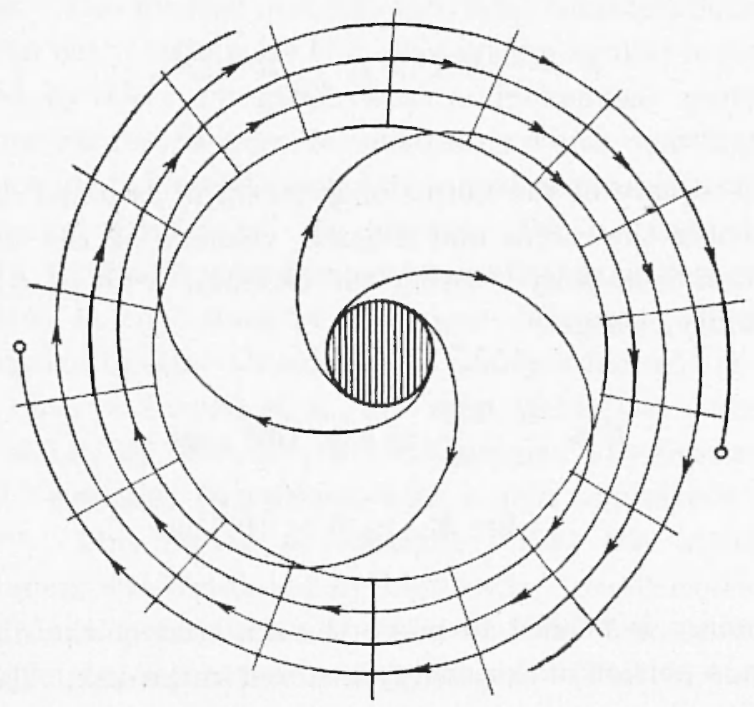

Figs 6

(after Hoyle, 1960).

The problem of distribution of angular momentum is not confined to the Solar system only. In fact, the same trouble also comes in, in different forms though, in the problems of the origin of double stars and more seriously in the formation of stars from an initial gaseous protoGalaxy. 
In the second case, however, the magnetic intensity $H$, and hence the magnetic pressure $H^{2} / 8 \pi$ steadily increases within the disk, and ultimately the disk may explode as energy will gradually be built up with the passage of time. These two cases can be distinguished as to whether the star possesses a deep convection zone or not. If it does we have the former case, otherwise the latter one. Basing on this criteria we have the representation of the first case in dwarf stars (the Sun is one such dwarf star), and the second one in stars like Pleione. Strure, Swings, Underhill, Merrill and others have observed spectroscopic peculiarities of Pleione which are explained by Crampin and Hoyle (1960) as due to the development and dissuption of a shell surrounding the main star.

The conservation of energy should share equal attention like the conservation of angular momentum paid above. The energy of rotation of the primitive solar condensation at the onset of instability according to Hoyle is:

$$
E=\frac{1}{2} M K^{2} \Omega^{2} \approx \frac{1}{20} R^{2} M \Omega^{2},
$$

when $K^{2}$, the square of the radius of gyration is assumed as $\sim 0,1 R^{2}$, and $R, \Omega$ denote the radius and angular velocity of the condensation at the onset of instability. Using the relation, $R^{3} Q^{-} \cong G M$ for rotational instability, we get:

$$
\begin{aligned}
E \approx \frac{1}{20} \frac{G M^{2}}{R} & \cong 5 \times 10^{45} \mathrm{ergs}, \\
\text { for } R & \cong 3 \times 10^{12} \mathrm{~cm} .
\end{aligned}
$$

Since energy is needed to move the material of the disk outward from the Sun, a portion of this energy is stored in the disk. This amounts to $\sim G M m / R$, where $m$ is the mass of the disk. Since $m \sim 10^{-2} M$, as reasonably assumed this is less than the original energy by a factor $\sim 5$. Thriefore, only a moderate portion of the energy is stored in the disk. Part of the rotational energy appears transformed into magnetic energy, and in its windings, as the rotation does work against the magnetic field. The dissipation of magnetic energy takes place through Sun-spots, flares etc. The abnormally intense $T$ Tanri stars which are of recent formations and give a way large reservoirs of internally stored magnetic 
energy through flares adds observational support to the fact that most of the Sun's initial energy was dissipated.

To reiterate what has been said before, as the planetary material acquired angular momentum it mored farther and farther away from the solar condensation. Materials of low volatility condensed out of the gas and were left behind as the main mass of gas mored outwards. The fact that the terrestrial planets (Mercury, Venus, Earth, Mars) are of small mass composed almost wholly of low rolatility materials on the inside of the system is due to this leaving behing of low volatility materials (Hoyle, 1960).

4. Recently, Fowler et al. (1962) studied the nuclear reactions at the early stages of the solar system. They considered the synthesis of $\mathrm{I}^{2}$, $\mathrm{Li}^{6}, \mathrm{Li}^{7}, \mathrm{~B}^{10}$, and $\mathrm{B}^{11}$, also of $\mathrm{C}^{13}$ and $\mathrm{N}^{15}$ by nuclear reactions due to high energy particles, mainly protons from Solar flares bombarding the planetesimals. The nuclear and astrophysical considerations of Fowler et al. reveal an early history for the solar system similar in some-ways to that depicted by Urey on geophysical and chemical grounds. They believe the nuclear results provide constraints which constitute the most direct evidence available concerning the physical conditions that existed at the beginning of origin of the planets. For instance, it has been found that $\mathrm{D}, \mathrm{Li}, \mathrm{Be}, \mathrm{B}$ were formed by spallation (disintegration) neutron irradiation in conditions of Hydrogen deficiency. The calculated deficiency relative to solar abundances is nearly a factor $4 \times 10^{3}$. Such a situation, observe Fowler et al., not only yields the correct relative abundances of $\mathrm{Li}^{6}, \mathrm{Li}^{7}, \mathrm{Be}^{9}, \mathrm{~B}^{10}, \mathrm{~B}^{11}$ but also, as a by product, explains the origin of terrestrial Deuterium, with a $D / H$ abundance ratio equal to $1.5 \times 10^{-4}$. This makes us conclude "that the material of the terrestrial planets was irradiated by high-energy particles from the Sun, at a stage of development when the material had become very largely, but not completely, separated from hydrogen" (Fowler etc.).

Astrophysical considerations requires that Hydrogen deficiency will arise, caused by the planetesimals falling out of the Hydrogen and Helium gas that so long carried them along, when the planetesimal radius $\Rightarrow 1 \mathrm{~m}$. Nuclear arguments that the efficiency of absorption of solar particles be not very small, as well as that not all planetary material be irradiated, otherwise nuclei such as $\mathrm{Gd}^{15}$ and $\mathrm{U}^{235}$ will be scoured out as their thermal neutron capture cross-sections are rery large, dictates the size of the planetesimals $\approx 10 \mathrm{~m}$ (Fowler et al. 1962). Thus Fowler et al. 
infer there were no large proto-planets as adrocated by Kuiper, in which Hydrogen was present in the normal solar proportion together with the notion that the Earth itself had erer a strongly reducing atmosphere composed of hydrogen.

During the formation of planetesimals there were aroording to them high concentrations of $\mathrm{H}_{2} \mathrm{O}, \mathrm{NH}_{3}, \mathrm{CH}_{4}$ in the surrounding reducing medium. Probably biologically interesting molecules were evolved at that stage before the formation of planets. Evidence can be adduced for the existence of complex organic compounds (Life ?) in carboncontaining meteorites cited by Brigrss and Urey.

Studies of the amount of extinct radioactivities should be consistent with the chain of physical and chemical events oceurring from the begining of the Solar-system. This work made by Cameron leads to the conclusion that the time required for the entire chain of events is not inconsistent with the interpretation of Xenon and the Silver isotopic anomalies in the meteorites due to extinct radioactivities left over from the process of nucleosynthesis preceding the formation of the solar system within our Galaxy. The evidence from the extinct radionctivities indicates that a short time (a few million years) passed between the start of condensation of Solar system from the interstellar medium and the formation and cooling of the meteorite parent bodies. On the problem connected with extinct radioactivities is the manner of production of the radioactive isotopes involved. Fowler, Greenstein and Hoyle (1961-62) have proposed that the extinct radioactivities were made by spallation and neutron processes in the planetesimals. Cameron (1962) however, thinks that their supposed process in unlikely, and considers the extinct radioactivities as surviving from a period of nucleosynthesis in the Galaxy which enriches the insterstellar medium with fresh radionctivities a relatively short time $\left(\approx 10^{\text {; }}\right.$ years $)$ before the formation of the Solar system. (The maximum rate of nucleo-synthesis may have occurred about 13 billion years ago. Clayton, 1964).

In fact, Cameron's work, though admitted by the Author himself to be crude, is one of the best ever written on this subject and should be consulted by interested readers. He deduces the following cosmochronology associated with the early history of the Solar-system. "If the main heat source required to melt Iron in meteorite parent bodies is $A \mathrm{l}^{26}$, the time interval between the start of condensation and the formation of the meteorite bodies is less than $6 \times 10^{6}$ years. From the anomalous Silver composition in Iron meteorites it is deduced that the time interval to the solidification of Iron in meteorite parent bodies 
is 2 to $4 \times 10^{7}$ years. From the anomalous Xenon composition of meteorites it is concluded that the time interval to the cessation of Xenon diffusion in the meteorite parent body is about $1.5 \times 10^{8}$ years".

In addition to the apparent shortness of time required for the formation of protostars and planets, as studied by Cameron, who also concludes that very massive binary companions can also condense out from very massive nebular disks which should accompany the process of star formations. The formation of binary companions will depend upon the nature of mass distribution controlled by the magnetic fields in the nebular disk. If they are formed, they will provide strong interference to planet formation.

In our above deliberations we have implicity assumed that the Sun as well as the planets were genetically connected with the primordial gas clond. Let us, however, ask ourselves the alternative question: could it be not that the Sun came near a wandering cloud and captured it subsequently? Hoyle, Bondi, Lyttleton and McCrie thought it possible for a passing star to seize matter from the surrounding nebula and get "rejuvenated", as it were, and that the star should move up along the curve of the main sequence of H-R. spectra-luminosity diagram. This process could occur, following them, if the star is rather massive and approaches the nebula very slowly. However, to all intents, this may be considered higly impossible. Each active star, as shown by G. Gurzadyan, repels the hydrogen dust by radiation pressure. V. Safronov has calculated that stars having a surface temperature of nearly $8000 \mathrm{~K}$ or higher repulse dusts against gravitation, and varions observations have ronfirmed it. So encounters of stars with particles of a nebula will generally be insignificant. The authors of "rejurenation" theort, however, believe that this can occur indirectly: by its gravity a star distorts the path of passing particles and they get concentrated in its rear part whence these particles collide with each other and after loosing their kinetic energy thereby, finally fall on the star. But even this can happen only if the star penetrated the nebula sufficiently slowly. But any outside star originally unconnected with a nebula must necessarily gain speed because of the gravity of the nebula. So we infer that when a star moves very slowly with respect to the nebula it must be genetically connected with it. Probably the star developed in the heart of the nebula and didn't find itself in it as an 'epilogue: due to accidental passing.

The next question which immediately suggests itself concerns the mode of origin of the gas cloud. The composition of the Solar system 
having many heavy and radioactive elements should offer a clue to their origin. Salpeter (1959) and Schmidt (1959) believe that most of the clements had genesis in the early stage of Galactic evolution. However, to explain the occurrence of all the extinct radioactivities, e.g. $\mathrm{Pu}^{239}, \mathrm{Pu}^{240}, \mathrm{Pu}^{242}, \mathrm{Pu}^{244}, \mathrm{Am}^{243}, \mathrm{Cm}^{246}, \mathrm{Cm}^{247}, \mathrm{Cm}^{248}, \mathrm{Cf}^{250}, \mathrm{Cf}^{251}, \mathrm{Cf}^{252}$ and $\mathrm{Fm}^{255}$, inferred from the isotopic composition study of their daughter products, $\mathrm{Th}^{232}, \mathrm{U}^{235}$ and $\mathrm{U}^{238}$ and of meteorites, it is presumably convenient to imagine (Cameron, 1962) that a second stage of element formation also took place just prior to the "becoming" of the Solar system. From the estimate of radiogenic $\mathrm{X} e^{129}$ in the atmosphere Kuroda and Manuel (1962) concludes that the time interval between the nucleosynthesis and the formation of the earth $\sim 2.4 \times 10^{\circ} \mathrm{yrs}$. The extinct radioactivities could not otherwise survive the long period between the birth of the galaxy $\left(\approx 20 \times 10^{9} \mathrm{yrs}\right)$ and the origin of the Sun and planets $\left(\approx 5\right.$ or $6 \times 10^{9}$ yrs. $)$. The heavy elements were formed in the nuclear ash's in the vast cosmic explosion of a Type II Supernovae. The debris of the explosion then enriched the interstellar gaseous medium with heavy metals from which the Solar system was ultimately born. The study of the relative abundances of heavy metals in Sun should also confirm (or otherwise) some astronomers belief that the Sun is probably a third generation star, having derived its matter from the ashes of other dead stars. Though the general abundance curves for elements in the Sun and the meteorites are very similar (Urey 1962) the low abundances of Iron and lead in the Sun (Aller 1959) may be due to their origin from different samples of nucleogenesis or due to some nuclear reactions or chemical fraction process (Urey 1962).

5. At present, the problem apparently related with the question of origin of the Solar system and which has sought a common goal for the various fields - general relativity, cosmology and geophysics is regarding the generalisation of Einstein's theory of gravitation. This extension, observes Dicke, brings the theory more nearly in accord with Mach's principle wich emphasizes that inertial effects should be associated with acceleration of matter relative to other matter, not relative to an absolute space. Out of Mach's principle evolved spectacular conglomeration of ideas called relativity.

This expansion of Einstein's field equations (Jordan, 1955) makes gravitational field composed of both scalar and the classical tensor fields. One fundamental property of the scalar field, known to very 
few physicists, according to Dike (1962) is that the mass of a particle interacting with a scalar field is a function of the scalar. For practical reasons the mass of a particle may be kept constant by a transformation of the basic units, and it is then seen that such transformations lead to the dependence of the gravitational constant upon the field scalar, and hence it is variable. By making use of elementary dimensional analysis and Mach's principle (that accelerations are determined by the distribution of distant matters without reference to the strength of the field), Dicke has shown that the gravitational constant $G$ is a function of the matter density of the universe. As the matter density of the universe is falling due to the general expansion, it may lead to the secular change of the locally measured valued of $G$. For further details on this question from the viewpoint of the physicists, the readers are referred to Dicke (1962), and Jordan, and also to Neumann, Edward Teller, Gamow, Ter Harr, Brill, Egyed, Ewing, Wilson, Heezen, etc., in connection with geophysical consequences of this liypothesis (known as Dirac's hypothesis). When the different constant, the relocity of light, Plank's constant and the mass of an elementary particle, say, pion are expressed dimension less in atomic units as unity, there is an apparent relationship between the gravitational constant, the inverse of the age of the universe $=t$, and the mean matter-density of the cosmos all being $\approx 10^{-10}$ (dimension less) $\left(^{*}\right)\left({ }^{58}\right)$. Dirac (1938) was apparently impressed by this relation and suggested that all the three parameters, including $G$ is a function of $t^{-1}$.

We may now set ourselves the problem: how the variable $G$ (assuming it is true) in fluenced the origin of the planetary systems out of the primordial chaos of the then new-born Galaxy, and also what effect are there on earth of the changing distribution of distant matter, the expansion of the universe being connected with decrease of $G$. Assuming that $G=C / t$, where $C$ is a constant and $t$ the age of the universe, Esyed (1960) has suggested a possible mechanism whereby the planets were formed from the equatorial region of the rapidly spinning primitive sun due to the interplay of the centrifugal force and gravity acceleration. When, a factor of $1 / r^{2}(r=$ radius of the sun) is neglected as it is very small, the two forces vary as $a /\left(r_{o}+a . t\right)$ and $B / t$ respectively where $r_{0}=$ the initial radius and $c$ and $B$ are constants. The two forces vary as shown in the Fig 7 . When the two forces become equal, a

(*) For the significance of large dimensionless quantities of the order of $10^{40}$, also known as cosmic numbers, one may refer to Harrison (1963). 
part of the mass, a ring, around the equator becomes weightless. Also the radius of this ring will increase in time according to the law $R=G t$. The increasing radius disturbs the equilibrium and the ring will eventual$1 y$ condense into a planetary body. Hence as the planet has formed from the equatorial ring its orbit coincides with the Sun's equator, as observed. This theory of Egyed as it stands is rudimentary and details need be worked out to explain the previously mentioned observed regularities of the Solar system.

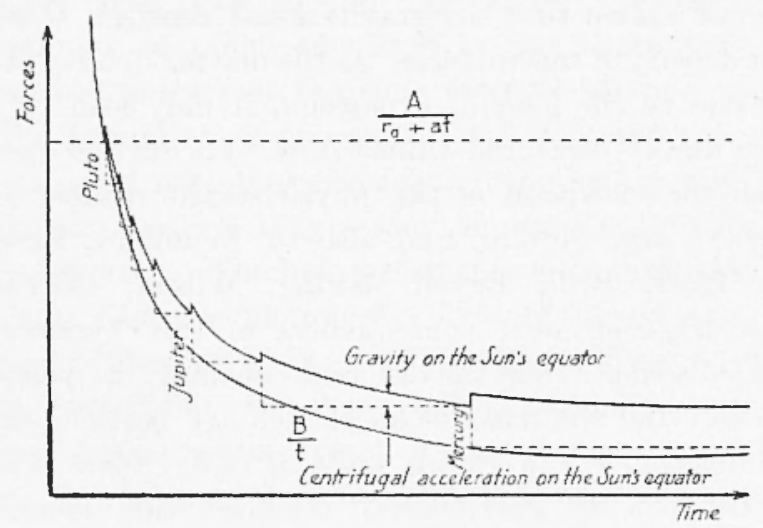

Fig. 7

(after Egyed, 1960.)

We shall now briefly discuss the imprints upon the Earth of decreasing gravitation. The first one striking the mind is the slow expansion of the Earth (*) though the mode of expansion is a little uncertain. The other areas where the effects should be sought are in the thermal history of the Earth and its magnetic fields. Studies of paleomagnetism and other geological evidence, have almust confirmed continental drift (**) (Fig. 8); however there is some doubt regarding the physical mechanism. The global system of tension cracks in all the oceans (Ewing, Heezen, Tharpe

(*) The suggestion that the Earth might be expanding was marle long before any plausible physical mechanism such as secular change in $G$ was postulated. Historically, the original irlea of expansion was offered by Mantovani at the end of the nineteenth century, to explain the similarity of the Atlantic coast lines. (L. EGyed, Nature, $\vee$. 197, No. 4872, p. 1059-69, 1963).

(**) A Royal Society of London discussion on continental drift was recently held in Iarch 20.21, 1964 and reported by prof. S. K. Runcorn in the Times Science Review, Summer, 1964. 

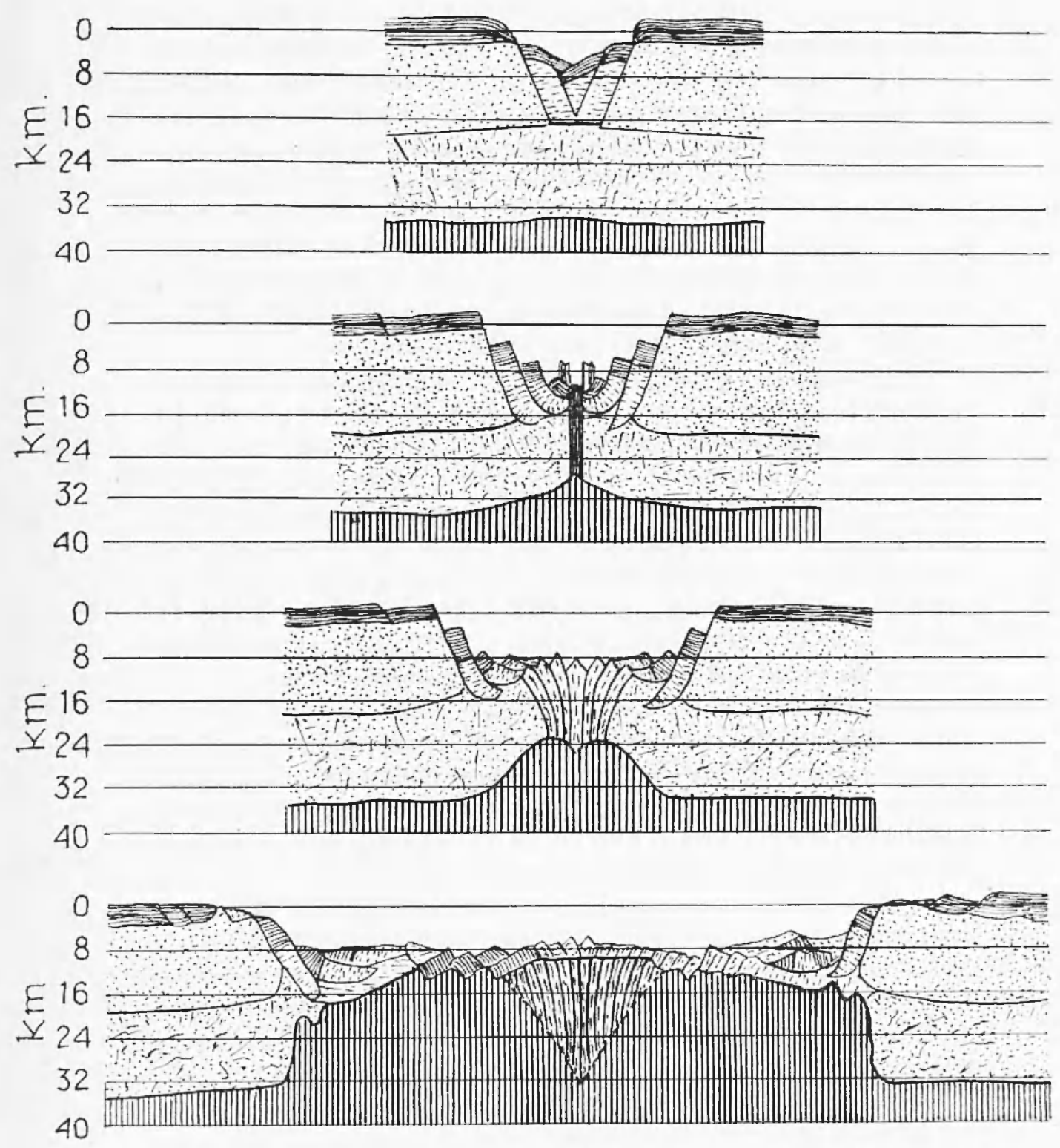

Fig. 8 - The growth of ocean fioors and drift of continents according to Heezen's "blown-up fotthall " theory. 
1960 Fig. 9) may suggest their genetic relation with a general expansion. While this may be qualitatively true, the Atlantic Ocean here presents a major problem. All evidences indieate that the Atlantic Ocean is recently formed in less than $200 \mathrm{~m}$ years. The expansion rate is nearly 300 times more than theoretically predicted on assuming $G$ varying 3 parts in $10^{11}$ parts per year. There may be a way out by assuming a large volume change of the Earth due to a phase change caused by a small change in G. However, evidences concerning Earth's rotation seems to rule out such possibility of large change of Earth's radius (Dicke 1962). It is hence possible that mantle convection (ursents have conspired with the slow expansion of the Earth to cause continents drifting.

The heat flow from the ocean bottom measured as $\approx 50 \mathrm{ergs} / \mathrm{cm}^{2} \mathrm{sec}$, posed a problem so long. Assuming a certain distribution of radioactive elements $\mathrm{U}$, Th, and $\mathrm{K}$, the calculated heat flow was too low. The discrepancy largly disappears with the introduction of a decreasing gravitation. This makes the pressure as well as the melting point of the mantle decrease, and under the assumption of steady convective transport heat will be originated in a cooling interior, and thus theory broadly supports the observation ( $\left.{ }^{27}\right)$. Also part of the heat required to produce core convection to explain Earth's magnetic field is conceivably generated by slow cooling due to weaking $G$. The other thermal problem is concerning paleoclimatology. Basing on the theory that the luminosity of the Sun varies with $G$, being proportional to 7.5 powers of $G$, the surface temperature of the Earth could be found out. Recomputation by Gamow of Edward Teller's original calculation shows a high temperature at the beginning of the Cambrian era. This points to high temperature genesis of life and leads to the trilobites and early mollusks playing in a very warm water, whether it could be so we leave it to the life-scientists for judgment. Moreover, such high average temperature makes pre-Cambrian or even permo-Carboniferons glaciation unlikely and fails completely to explain the repeated periods of intense glaciation the Earth experienced. Jordan (1962) however differs from the view presented above regarding the problem presented by paleoclimate.

Besides Earth, other celestial bodies may also provide empirical evidences of weakening $G$, e.g. slow incrase in the radii of their orbits by double stars, the explanation of which eluded Jeans (Jondon 1955). Like the Earth, secular change in $G$ should cause slow expansion of the Moon, presumably by the extrusion of magma. The Moon is a better object to study for it still bears the 'fossil' records of its history untouched 
by geological changes wrought by the sea and the wind. An interesting problem of very near-future space research should be to land on Moon and verify if the craters are filled with basalts. These igneous rocks, we presume, would be erupted along the fractures opened up due to expansion of the Moon due to the diminishing G. Evidence of tension cracks caused by the IIoon's expansion should also be searched in photographs taken from satellites orbiting the Moon.

A further note on Moon. As regards its internal structure and formation of its tectonies, viz., lunar craters and ring mountains, Hedervari made calculations assuming that release of gravitational pressure caused evolution in the internal structure of the Hoon. For example, the Gutenberg discontinuity was much shallower at an early epoch than at present the discontinuity surface indicating phase change between metallic core and non-metallic mantle, going down continuously with the passage of time. The secular lessening of pressure ultimately lead to the ranishing of the core and the Moon became homogeneous as its present moment of inertia $\approx 0.4$ suggests. Furthermore, Hedervari pointed out that the energy produced by gravitational pressure release is quite sufficient (in fact, about $10^{\text {s }}$ times more than needed) to produce the surface tectonics.

If Dirac's hypothesis of secular variation of $G$ is correct, one important consequence is that the ages of stars inferred from stellar evolution would be incorrect. For example, assuming the stars radiate energy which is proportional to $G^{7}$, the 15 billion years old stars of $\mathrm{O}$. C. Wilson should then become only 4.2 billion years (Dicke). Thus an important result emerges which Dicke considers his main success of the theory of variable 'constant' of gravitation - that he has brought the age of an evolutionary universe, the ages of the oldest stars in the Galaxy, the age of the Galaxy and the age of the heavy elements, into close agreement. Dicke's cosmology, however, is the complete opposite of the steady-state model of the Universe. Dicke in deriving his cosmology incorporated IIach's principle (a completely empty space cannot have any physical properties of inertia and geometry), and one of the consequences followed was the concept of time-varying ' constant' of gravitation. It may be left an open question whether Iach's principle could in fact be incorporated into the theory of gravitation. To quote Otto Heckmann (Dec. 1963).

"Personally I am not sure whether the attempts to incorporate Mach's principle into the theory of gravitation are really justified because the idea that matter has a reality but empty space-time has not seemed 


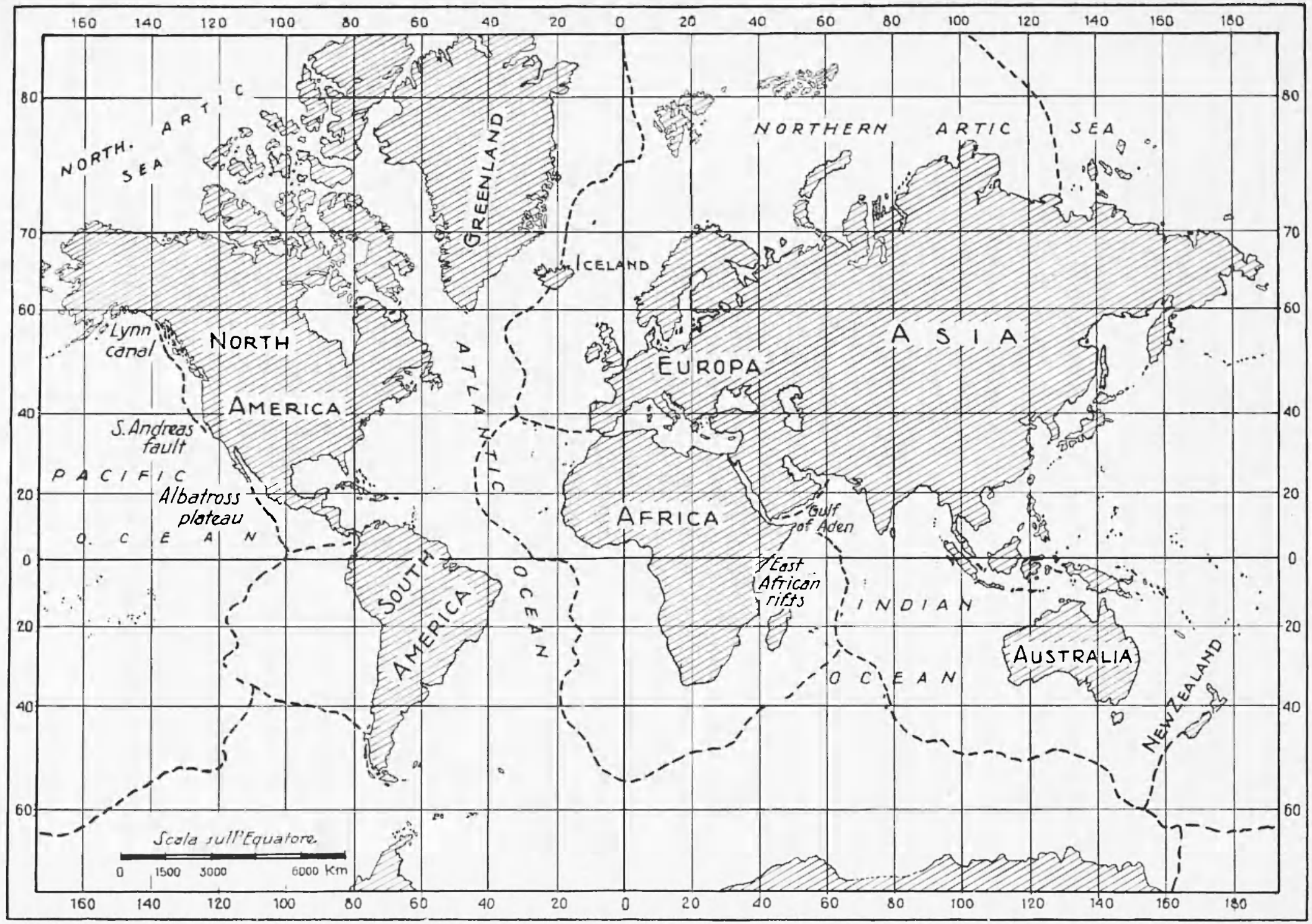

Fig. 9 - The system of miloceanic rifts, after Maurice Ewing \& Others. 
to me somewhat old-fashioned at a time when fields are used to represent matter. Why shouldn't the field of space-time represent inertia?".

In our above discussions we have assumed validity of Dirac's hypothesis and endeavoured to discover empirical facts from the Earth and extra-terrestrial bodies. The implications of this hypothesis for earthscience, physies and cosmology are apparently not little. One further observational support of the variable $G$ is based on the theme that the meteorites were warmer at the begining of the solar system, leading to loss of argon from them. Peebles and Dicke (1962) have shown from the observed $\pi-A r$ ages of Meteorites that the maximum decrease of gravitational constant is one in $10^{10} \mathrm{per}$ year. It may also be noted that an important problem for space research should be to verify the hypothesis by accurate satellite measurements of the Farth's gravity field at different times. A comparison between an atomic measure of time provided by an earth-bound atomic clock with the gravitational measure of time provided by an artificial earth-satellite would serve to show, suggests R. H. Dicke, the acceleration within a relatively short time of a year. To quote Dicke:

"If the age of the Universe is taken to be $10^{10}$ years, then the gain of atomic over inertial time will be 1 part in $10^{10}$ in 1 year. If the atomic standard is reliable over long periods (months) to 1 part in $10^{11}$, as it is hoped, and if the satellite period can be determined to 1 part in $10^{11} \mathrm{in}$, say, 1 yeall (an accuracy that appears to be attainable through the use of the most refined modern techniques...), then the effect of a timevarying gravitational constant should be readily detectable".

6. Form the foregoing discussions it appears the monistic theories will succeed better than the dualistic ones in explaining the origin of the Solar system. Te should do well to reflect on the magnificant insignificance of the chance of colision as provoked in all catastrophic specoulations by studying Jeans' calculation: with the present structure of the Milky-Way the passage of any star close to our Sun is one in $10^{17}$ stars per year. As the Galaxy is only $10^{10}$ years old, one such encounter can happen only once in $10^{7}$ stals throughout its existence. However, any theory must satisfy the various geophysical, chemical, astrophysical and nuclear boundary conditions and ground rules. Looking back we see a wake of discarded theories left behind and to this extent we probably have progressed, for we know what the Unirerse is not like. It is possible that the judgment between one theory and the other will 
ultimately rest on aesthetic preferences; for instance, the Copernican system of the motions of hearenly bodies is accepted over Ptolemy's complicated epicycles by virtue of the former's nave simplicity and mathematical elegance. To sum up, the whole problem of the origin of Solar system, like the question of the creation of the Universe itself, for its complexity may call to one's mind, Sir Winston Churchill's famous phrase, "a riddle, wrapped in a mystery, inside an enigma".

\section{ACKNDWIEDGHFNT.}

I heartily express my gratitude to Prof. Pietro Caloi, National Institute of Geophysics, Rome, Italy, for kindly taking interest in my work and correcting it. I am grateful to Dr. C. Gilbert, Dept. of Mathematics, King's College, Newcastle-upon-Tyne, University of Durham for kindly sending me the calculations on Moon (by Hedervari) and suggesting some idleas in the work described in section 5. I am thankful to Mr. S. N. Sengupta, Director, Mr. Subrata Ray, Sr. Geophysicist (both in Oil \& Natural Gas Commission, Debra Dun), Mr. A. Rahman, Officer on Special Duty, Council of Scientific \& Industrial Research, New Delhi, and Prof. P. K. Bhattacharjee of Indian Institute of Technology, Kharagpur, for their encouragement.

\section{BIBIIOGRAPII}

(1) Alrvex H., On the origin of the Solar System. Clarendon Press, Oxford (1954).

(2) BERLAGE H.P., The basic scheme of any planetary or satellite system corrected and reanalysed. I \& II Koninkl. Nederl. Akademic Van Wetenschappen, Ansterdam. Series B, 62, 1 (1959).

(3) BerLAge H. P., On aceretional instability, the state, leading to the transformation of a gaseons disc, rotating in Quasi-Steady motion round a massive centre, into a set of concentric rings of particulate matter. Koninlkl. Nederl. Akademic Van Wetenschappen, Amsterdam, Series B, 65, 3, 199220 (1962).

(") BIRD J. F., Thesis on " Massive condensations in Interstellar Matter and stellar Associations, ,. Cornell University, 1958.

(5) Cayrerox A. G. W., The formation of the sun and Planets in ICARUS, 1, I (1962).

( $\left.{ }^{8}\right)$ Chamberdin T. C., The origin of the Earth. University of Chicago Press, 1924. 
(7) Crampin F. and IIoYle F., Problems concerning Pleione, in Monthly notices of the Royal Astronomical Society, 120, 1, 33-42 (1960).

$\left({ }^{8}\right)$ EGred L., Dirac's cosmology and the origin of the solar system. "Nature", 186, $4725,621-622(1960)$.

$\left({ }^{9}\right)$ Fowler W. A., Greexsteix J. L. and Horte Fred, Nucleosyntheis during the early history of the Solar System. "The Geophysical Journal of the Royal Astronomical society" London (1962).

${ }^{(10)}$ Ganow G., The creation of the Cniverse Mentor Book 1959.

(11) Jordan P., Geophysical consequences of Dirac's hypothesis, in "Rev. of Mod. Phy.", 34, 4, 596-600 (1962).

${ }^{(12)}$ Joxes II. S., The origin of the Solar system:, in "Physies and Chemistry of the Earth", 1, Pergamon Press (1956).

$\left({ }^{13}\right)$ KanT I., Allgemeine Naturgeschichte and T'heorie Des IImmels. 1755.

$\left({ }^{14}\right)$ Kopal, ZDExeK, Close binary systems. International Astrophysieal se. ries, V, Chapman and Hall Ltd., 1959.

$\left.{ }^{15}\right)$ Kuipin G. P., On the origin of the Solar System, in "Astrophysies topical symposinm" Ed. J. A. Hynek, Magraw IIill., 1951.

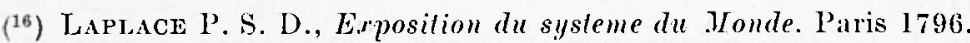

(17) Ovendex M. W., The origin of the Snlar System Discovery. Norwich, England, 1960.

${ }^{18}$ ) Oparin A. and Fesenkov V., The Universe. Foreign Languages Publish. ing House, Moseow, 1960.

(19) Pikelxer S., Physics of Interstellar Space. Foreign Ianguage Publishing House, Joscow (in Engrlish).

$\left({ }^{20}\right)$ Scum mo O. J., A theory of Earth's origin. Foreign Languages Publishing House, Moscow, 1958.

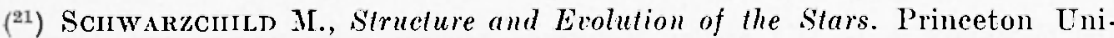
versity Press. New Jersey, 1958.

${ }^{(22)}$ Tenr IIAAR D., Studies on the origin of Solar System: thesis. Leiden, 1948 .

$\left({ }^{23}\right)$ Uney H. C., Boundary conditions for the origin of the Solar System, in "Physies and ehemistry of the Earth", II. Pergamon Press (1957).

${ }^{(24)}$ Urer II. C., The Planets: their Origin and Derelopment. Yale University Press, New Haven, 1952.

${ }_{(25)}$ Urex II. C., Proceedings of the Leningrad. "Symposium on Moon" (1960).

${ }^{(26)}$ Werzsacker (. Von, The II istory of Vature. Routledge \& Kegan Paul, London, 1950 .

Further ildditions.

${ }^{(27)}$ DICKE R. II., The Earth and Cosmology, in "Science", 138. 3541, 653. $66+(1962)$.

${ }^{(28)}$ IIorte F., On the Origin of Solar Nebula, in "Quarterly Jour of Royal Astr. Soc.”, I. 28-55 (1960). 
$\left.{ }^{(29}\right)$ EGYED L., On the origin and Constitution of the upper part of the earths montle, in "Sonderdruck aus der Geologischen Rundschau" 50, 251$258(1960)$.

(30) JORDAx P., Remarls about Ambarzumian's conception of pre-stellar matter, in "Recent development in General Relativity", Pergamon Press, 1962.

(31) Jordax P., Schwerhraft and Weltall. Vieweg, Brumswick, Germany, 1955.

$\left.{ }^{32}\right)$ JoRDax P., Empirical confirmalion of Dirac's hypothesis of diminishing Gravitation. in "Recent developments in General Relativity". Pergamon Press, 1962.

(33) Jordan P., Zum. Problem der Expansion, in "Naturwiseenschaften", 11, $417-425(1961)$.

(34) PEebies J. \& Dicke R. H., The Temperature of Meteorites and Dirac's Cosmology and Mach's Principle, in "Jour. Geop. Res.", 67, 10, $4063-4070(1962)$.

(35) Teluer E., On the change of physical constants, "Pliys. Rev." 73, 801 (1948).

$\left.{ }^{(36}\right)$ Wilsox J. T., Some consequences of expansion of the earth, "Nature", 181. $880(1960)$.

37) Dikac P. A. M., A new basis for cosmology, "Proc. Roy. Soc. London", A. 165,199 (1938).

${ }^{(38)}$ Potapov I. I., On the problem of the origin of the earth (abstract) (in Russian). Vyssh. Teheb. Zavedeniy l zv, "Geologiya i Razwedka", 1. 3.18 (1962).

$\left({ }^{30}\right)$ LEVIx B., The origin of earth and planets. 3rd. edition, Foreign languages Prublishing House. Moscow,

(40) Canerox A. G. W., Stellar evolution, Nuclear Astrophysies and $N u c l e o-$ genesis. Atomic Energy Commission Chalk River Report, Ontario, 1961.

(11) Kurona P. K. \& Maxver, O. K., On the chronology of the formation of the solar System. 1. Radiogenic Xenon 1:\%, in The Earth's atmosphere. "J. of Geop. Res.", 67, 12, 4859-4862 (1962).

${ }^{42}$ ) Kuroda P. K. \& Crouch W. H. (Jr.), On the chronology of the formation of the Solar System. 2. Iodine in Terrestrial rocks and the Xenon 129/136 formation internal of the earth, "J. of Geophysics Res.", 67, 12, 4863-4866, (1962).

${ }^{(43)}$ Kurodi P. K., Nuclear fission in the early history of the earth. "Nature" 187. $36-38(1960)$.

(44) Kuroda P. K., The time interval between the mueleosynthesis and formalion of the earth. "Geochimica et. cosmochimica Acta", 24. 40-47 (1961).

(45) UREY HAROLD C., Evidence regarding the origin of the earth, in "Geochimica et Cosmochimica Acta", 26. 1 -13 (1962).

${ }^{(46)}$ Aller L. H., Abundances of elements in the sun and stars. Handbuch der Physik, 51, 324 (1959). 
THE EARTI IN SPACE: AN ESSAY ON THE ORIGIN OF THE SOLAR SYSTEN 447

$\left.{ }^{(47}\right)$ Whipple, F. L., The dust cloud hypothesis in The New Astronoms. Editors of Scientific American, 1948.

${ }^{(8)}$ Egred L., The expansion of the earth in connection with its origin and evolution, "Geophysica ", 7, 13.22 (1960).

(19) Camerox A. G. W., The origin of the elements in Physics and Chemistry of the earth. 3, Pergamon Press, 1959.

(50) Ganow G., Turbulance in Space, in "Sc. Amer.", June 1952.

(51) Hedervari, P., On the energy necessary to produce lunar eraters and ring-mountains, in "Hungarian Physical Review, (Mayar Fizikai Folyoirat)" of the Acardemy of Aciences, 1961/64.

(52) Henervari, P., The Gravity "Constant" as the Function of the Time and the Internal structure of the Moon, in "Hungarian Physical Review ", 4 sept. 1960. Budlapest. No. 4.

(53) Drcke, R. H.: Gracitation - an Enigma, "American Scientist", v. 47, no. 1, p. 25-40, (1959).

(54) Dicke, R. H., The Nature of Gravitation. p. 91-118.

(55) Cuarox, D. D., Chronology of the Galary, "Science" v. 143, No. 3612, p. $1281.1286(1964)$.

(56) Huckmaxi, O., Aspects of Modern Cosmology (unpublished), lecture delivered at the annual meeting of the American Association for the Advancement of Science, Cleveland. Dec. 1963.

(57) Girbert, C., Dirac's Cosmolog!y and the Gieneral Theory of Relativity, "Mon. Not. Roy Astro. Soc.", v. 116 no. 6, p. 684-690, (1956), also Dirac's Cosmology, "Nature", V. 192, No. 4797, p. 57, Oct. 7, 1961.

(58) Harrisox, E.R., Cosmie Numbers, "Nature ", v. 197, no. 4874, p. 1257-59, (1963). 